\title{
The Holonomic Rank of the Fisher-Bingham System of Differential Equations
}

\author{
Tamio Koyama*, Hiromasa Nakayama*, \\ Kenta Nishiyama ${ }^{\dagger}$, Nobuki Takayama*
}

February 18, 2013

Abstract. The Fisher-Bingham system is a system of linear partial differential equations satisfied by the Fisher-Bingham integral for the $n$-dimensional sphere $S^{n}$. The system is given in [4, Theorem 2] and it is shown that it is a holonomic system [1]. We show that the holonomic rank of the system is equal to $2 n+2$.

Keywords: Fisher-Bingham distribution, holonomic rank, Gröbner basis

\section{Introduction}

Let $x=\left(x_{i j}\right)$ and $y=\left(y_{i}\right)$ be parameters such that $x_{i j}=x_{j i}$ for $i \neq j$. Let $Z$ be a function, which is the normalization constant of the Fisher-Bingham distribution, defined as

$$
Z(x, y, r)=\int_{S^{n}(r)} \exp \left(\sum_{1 \leq i \leq j \leq n+1} x_{i j} t_{i} t_{j}+\sum_{i=1}^{n+1} y_{i} t_{i}\right)|d t|
$$

where $S^{n}(r)=\left\{\left(t_{1}, \ldots, t_{n+1}\right) \mid \sum_{i=1}^{n+1} t_{i}^{2}=r^{2}, r>0\right\}$ is the $n$-dimensional sphere and $|d t|$ denotes the Haar measure on the sphere.

Let $D$ be the Weyl algebra

$$
D=\mathbf{C}\left\langle x_{i j}, y_{k}, r, \partial_{i j}, \partial_{k}, \partial_{r} \mid 1 \leq i \leq j \leq n+1,1 \leq k \leq n+1\right\rangle
$$

where $\partial_{i j}=\partial / \partial x_{i j}, \partial_{k}=\partial / \partial y_{k}$ and $\partial_{r}=\partial / \partial r$. It is shown in [1] and 4] that the normalization constant (11) of the Fisher-Bingham distribution is a holonomic function in $x, y, r$ and consequently it is annihilated by the following

\footnotetext{
* Department of Mathematics, Kobe University and JST CREST Hibi team

${ }^{\dagger}$ Graduate School of Information Science and Technology, Osaka University and JST CREST Hibi team
} 
holonomic ideal $I$ in $D$ generated by the following operators in $D$ :

$$
\begin{aligned}
& \partial_{i j}-\partial_{i} \partial_{j}, \\
& \begin{array}{l}
\sum_{i=1}^{n+1} \partial_{i}^{2}- \\
x_{i j}^{2}
\end{array} \\
& \quad+2\left(x_{j j}-x_{i i}\right) \partial_{i} \partial_{j}-x_{i j} \partial_{j}^{2} \\
& \quad+\sum_{s \neq i, j}\left(x_{s j} \partial_{i} \partial_{s}-x_{i s} \partial_{j} \partial_{s}\right)+y_{j} \partial_{i}-y_{i} \partial_{j}, \\
& r \partial_{r}-2 \sum_{i \leq j} x_{i j} \partial_{i} \partial_{j}-\sum_{i} y_{i} \partial_{i}-n .
\end{aligned}
$$

We call the system of differential equations defined by $I$ the Fisher-Bingham system.

For a left ideal $J$ in $D$, the holonomic rank of $J$ is defined as the dimension of the $K=\mathbf{C}\left(x_{i j}, y_{k}, r \mid 1 \leq i \leq j \leq n+1,1 \leq k \leq n+1\right)$ vector space $K\left\langle\partial_{i j}, \partial_{k}, \partial_{r}\right\rangle / K\left\langle\partial_{i j}, \partial_{k}, \partial_{r}\right\rangle J$. The rank is denoted by $\operatorname{rank}(J)$. When $J$ is a holonomic ideal, the rank is finite. The holonomic rank agrees with the dimension of the holomorphic solutions of the associated system of linear partial differential equations at generic points and with the size of the Pfaffian equation associated to $J$. As to general facts on the holonomic rank, we refer to, e.g., the chapters 1 and 2 of $[\underline{6}$. The holonomic rank is a fundamental invariant of the $D$-module $D / J$ and there are several attractive studies on holonomic ranks. For example, Miller, Matusevich and Walther studied holonomic ranks of $A$-hypergeometric systems by introducing a new homological method [3].

We are interested in the holonomic rank of the Fisher-Bingham system $I$. We prove the following theorem in this paper.

\section{Theorem 1}

$$
\operatorname{rank}(I)=2 n+2
$$

In [4, we proposed a new method in the statistical inference which is called the holonomic gradient descent. The method utilizes a holonomic system of linear partial differential equations associated to the normalization constant. The complexity of the method depends on the holonomic rank and correctness of the method are proved by utilizing the holonomic rank. In the case of the Fisher-Bingham distribution, which is the most fundamental distribution in the directional statistics, Theorem 1 is applied in [2, which gives a generalization of the result in 4 shown with a help of a computer program. Our method to prove the theorem is the Gröbner deformation to the direction $(-w, w)$, which is discussed in [6] for $A$-hypergeometric systems, and a determination of Gröbner bases by hand with adding several slack variables which do not change the holonomic rank. 


\section{The Rank of the Diagonal System}

When the matrix $x$ is diagonal, the normalization constant $Z$ satisfies a system of linear partial differential equations for the variables $x_{i i}, y_{k}, r$. Let $\tilde{I}$ be the left ideal in $D$ generated by

$$
\begin{aligned}
& A_{i}=\partial_{i i}-\partial_{i}^{2} \quad(1 \leq i \leq n+1), \\
& B=\sum_{i=1}^{n+1} \partial_{i}^{2}-r^{2} \\
& C_{i j}=2\left(x_{i i}-x_{j j}\right) \partial_{i} \partial_{j}+y_{i} \partial_{j}-y_{j} \partial_{i} \quad(1 \leq i<j \leq n+1), \\
& E=r \partial_{r}-2 \sum_{i=1}^{n+1} x_{i i} \partial_{i}^{2}-\sum_{i=1}^{n+1} y_{i} \partial_{i}-n
\end{aligned}
$$

and $\partial_{i j}, i \neq j$. The ideal $\tilde{I}$ annihilates the function $Z$ restricted to the diagonal of $x$ [2].

Theorem 2 The holonomic rank of $\tilde{I}$ is $2 n+2$.

Our proof of the theorem reduces to the proof of the following proposition.

Proposition 1 Let $R$ be the ring of differential operators with rational function coefficients

$$
R=\mathbf{C}\left(x_{11}, \ldots, x_{n+1 n+1}, y_{1}, \ldots, y_{n+1}, r\right)\left\langle\partial_{11}, \ldots, \partial_{n+1 n+1}, \partial_{1}, \ldots, \partial_{n+1}, \partial_{r}\right\rangle .
$$

Let $R \tilde{I}$ be the left ideal of $R$ generated by $A_{i}, B, C_{i j}, E$. Let $<$ be the term order on $R$ which is the block order with $\partial_{r} \gg\left\{\partial_{i i}\right\} \gg\left\{\partial_{j}\right\}$. The order of the block $\left\{\partial_{i i}\right\}$ is the graded lexicographic order with $\partial_{11}>\cdots>\partial_{n+1 n+1}$ and that of the block $\left\{\partial_{i}\right\}$ is the graded lexicographic order with $\partial_{1}>\cdots>\partial_{n+1}$. A Gröbner basis of RI with respect to the term order $<$ is

$$
\begin{aligned}
& A_{i}=\partial_{i i}-\partial_{i}^{2} \quad(i=1, \ldots, n+1), \\
& B=\sum_{i=1}^{n+1} \partial_{i}^{2}-r^{2}, \\
& C_{i j}=2\left(x_{i i}-x_{j j}\right) \partial_{i} \partial_{j}+y_{i} \partial_{j}-y_{j} \partial_{i} \quad(1 \leq i<j \leq n+1), \\
& \left(\text { We put } a_{i j}=2\left(x_{i i}-x_{j j}\right), F_{i j}=y_{i} \partial_{j}-y_{j} \partial_{i}, C_{i j}=a_{i j} \partial_{i} \partial_{j}+F_{i j}\right), \\
& D_{k}=\partial_{k} B-\partial_{1} a_{1 k}^{-1} C_{1 k}-\cdots-\partial_{k-1} a_{k-1 k}^{-1} C_{k-1 k} \quad(k=1, \ldots, n+1), \\
& E=r \partial_{r}-2 \sum_{i=1}^{n+1} x_{i i} \partial_{i}^{2}-\sum_{i=1}^{n+1} y_{i} \partial_{i}-n .
\end{aligned}
$$

The initial monomials of the Gröbner basis are

$$
\begin{aligned}
& \operatorname{in}_{<}\left(A_{i}\right)=\operatorname{in}_{<}\left(\partial_{i i}\right), \operatorname{in}_{<}(B)=\operatorname{in}_{<}\left(\partial_{1}\right)^{2}, \operatorname{in}_{<}\left(C_{i j}\right)=\operatorname{in}_{<}\left(\partial_{i}\right) \operatorname{in}_{<}\left(\partial_{j}\right), \\
& \operatorname{in}_{<}\left(D_{k}\right)=\operatorname{in}_{<}\left(\partial_{k}\right)^{3}, \operatorname{in}_{<}(E)=\operatorname{in}_{<}\left(\partial_{r}\right) .
\end{aligned}
$$


Here, the initial monomial $\operatorname{in}_{<}\left(c(x, y, r) \partial_{r}^{\gamma} \prod \partial_{i i}^{\alpha_{i i}} \prod \partial_{k}^{\beta_{k}}\right)$ is defined as the element $c(x, y, r) \iota^{\gamma} \prod \xi_{i i}^{\alpha_{i i}} \prod \eta_{k}^{\beta_{k}}$ in the polynomial ring with rational function coefficients $\mathbf{C}(x, y, r)\left[\xi_{11}, \ldots, \xi_{n+1 n+1}, \eta_{1}, \ldots, \eta_{n+1}, \iota\right]$ (see, e.g., 6, Chapter 1]).

By the proposition, the standard monomials of the quotient ring $R / R \tilde{I}$ are $1, \partial_{1}, \partial_{2}, \partial_{2}^{2}, \ldots, \partial_{n+1}, \partial_{n+1}^{2}$. Therefore, the holonomic rank of $\tilde{I}$ is $2 n+2$ (Theorem 21). Let us prove the proposition.

Since $D_{k}$ is expressed by $B, C_{1 k}, \ldots, C_{k-1 k}$, the operator $D_{k}$ is the element in $R \tilde{I}$. In order to prove Proposition 1, we will show that any $S$-pair for $A_{i}, B, C_{i j}, D_{k}, E$ is reduced to 0 by $A_{i}, B, C_{i j}, D_{k}, E$. The following lemmas are proved by straight forward calculations.

Lemma 1 Let $P$ and $Q$ be elements in $R$. If the initial monomials are coprime, i.e., $\operatorname{gcd}\left(\operatorname{in}_{<}(P), \operatorname{in}_{<}(Q)\right)=1$, then the $S$-pair $S(P, Q)$ is reduced to $[P, Q]$ by $P$ and $Q$ (we denote the reduction by $S(P, Q) \underset{P, Q}{\longrightarrow^{*}}[P, Q]$ ), where $[P, Q]$ is the commutator of $P$ and $Q$. In particular, when $[P, Q]=0$, the $S$-pair $S(P, Q)$ is reduced to 0 .

Lemma 2 We have

$$
\begin{gathered}
{\left[A_{p}, C_{i j}\right]=0,} \\
{\left[B, C_{i j}\right]=0,} \\
{\left[C_{i j}, C_{j k}\right]=C_{i k},\left[C_{i j}, C_{i k}\right]=-C_{j k},\left[C_{i k}, C_{j k}\right]=-C_{i j} \quad(i<j<k),} \\
{\left[C_{i j}, C_{p q}\right]=0 \quad(\{i, j\} \cap\{p, q\}=\emptyset) .}
\end{gathered}
$$

Lemma 3 We have

$$
\begin{gathered}
{\left[D_{i}, A_{j}\right]= \begin{cases}0 & (i<j) \\
2 a_{j i}^{-2} \partial_{j} C_{j i} & (i>j), \\
\sum_{l=1}^{i-1} 2 a_{l i}^{-2} \partial_{l} C_{l i} & (i=j)\end{cases} } \\
{\left[D_{i}, B\right]=0,}
\end{gathered}
$$

When $i, j \neq k$, we obtain

$$
\left[C_{i j}, D_{k}\right]=\left\{\begin{array}{ll}
0 & (k-1<i) \\
0 & (j \leq k-1) \\
-a_{i k}^{-1}\left[C_{i j}, \partial_{i} C_{i k}\right]=a_{i k}^{-1}\left(\partial_{i} C_{j k}+\partial_{j} C_{i k}\right) & (i \leq k-1<j)
\end{array} .\right.
$$

Lemma 4 We have

$$
\begin{aligned}
& {\left[A_{i}, E\right]=0} \\
& {[B, E]=-2 B} \\
& {\left[C_{i j}, E\right]=0} \\
& {\left[D_{i}, E\right]=-3 D_{i}-2 \sum_{k=1}^{i-1} a_{k i}^{-1} \partial_{k} C_{k i}}
\end{aligned}
$$


Proof of Proposition [1. We prove that any $S$-pair for $A_{i}, B, C_{i j}, D_{k}, E$ is reduced to 0 by $A_{i}, B, C_{i j}, D_{k}, E$.

$S$-pairs of $A_{i}$ and $A_{j}, B, C_{i j}$. The initial monomials are coprime, and the elements commute. By Lemma 1] we obtain

$$
\begin{aligned}
& S\left(A_{i}, A_{j}\right) \longrightarrow^{*} 0, \\
& S\left(A_{i}, B\right) \longrightarrow^{*} 0, \\
& S\left(A_{i}, C_{j k}\right) \longrightarrow^{*} 0 .
\end{aligned}
$$

$S$-pair of $B$ and $C_{i j}$. When $i>1$, the initial monomials $\operatorname{in}_{<}(B)=\operatorname{in}_{<}\left(\partial_{1}\right)^{2}$, in $_{<}\left(C_{i j}\right)=$ in $_{<}\left(\partial_{i}\right)$ in $_{<}\left(\partial_{j}\right)$ are coprime. Operators $B$ and $C_{i j}$ commute by Lemma 2. By Lemma 1 we have $S\left(B, C_{i j}\right) \longrightarrow^{*} 0$.

When $i=1$, the initial monomials $\operatorname{in}_{<}(B)=\operatorname{in}_{<}\left(\partial_{1}\right)^{2}, \operatorname{in}_{<}\left(C_{1 j}\right)=\operatorname{in}_{<}\left(\partial_{1}\right) \operatorname{in}_{<}\left(\partial_{j}\right)$ are not coprime. We obtain the following reduction sequence of the $S$-pair:

$$
\begin{aligned}
& S\left(B, C_{1 j}\right)=a_{1 j} \partial_{j} B-\partial_{1} C_{1 j} \\
& =a_{1 j}\left(\partial_{j} \partial_{2}^{2}+\cdots+\partial_{j}^{3}+\cdots+\partial_{j} \partial_{n+1}^{2}-\partial_{j} r^{2}\right)-\partial_{1} F_{1 j} \\
& =a_{1 j}\left(\left(\underline{\partial_{j} \partial_{2}^{2}}+\cdots+\partial_{j}^{3}+\cdots+\partial_{j} \partial_{n+1}^{2}-\partial_{j} r^{2}\right)-\partial_{1} a_{1 j}^{-1} F_{1 j}\right) \\
& {\overrightarrow{C_{2 j}}}^{*} a_{1 j}\left(\left(\underline{\partial_{j} \partial_{3}^{2}}+\cdots+\partial_{j}^{3}+\cdots+\partial_{j} \partial_{n+1}^{2}-\partial_{j} r^{2}\right)-\partial_{1} a_{1 j}^{-1} F_{1 j}-\partial_{2} a_{2 j}^{-1} F_{2 j}\right) \\
& {\overrightarrow{C_{3 j}}}^{*} \cdots{\overrightarrow{C_{j-1 j}}}^{*} a_{1 j} D_{j} \underset{D_{j}}{\longrightarrow} 0 \text {. }
\end{aligned}
$$

$S$-pair of $C_{i j}$ and $C_{k l}(\{i, j\} \cap\{k, l\}=\emptyset)$. The initial monomials $\operatorname{in}_{<}\left(C_{i j}\right)=$ $\operatorname{in}_{<}\left(\partial_{i}\right) \operatorname{in}_{<}\left(\partial_{j}\right), \operatorname{in}_{<}\left(C_{k l}\right)=\operatorname{in}_{<}\left(\partial_{k}\right) \operatorname{in}_{<}\left(\partial_{l}\right)$ are coprime. Operators $C_{i j}$ and $C_{k l}$ commute by Lemma 2. By Lemma 1, we obtain

$$
S\left(C_{i j}, C_{k l}\right){\underset{C_{i j}, C_{k l}}{\longrightarrow}}^{*} 0 .
$$

$S$-pair of $C_{i j}$ and $C_{j k}(i<j<k)$. We have the following reduction sequence of the $S$-pair:

$$
\begin{gathered}
S\left(C_{i j}, C_{j k}\right)=a_{j k} \partial_{k} C_{i j}-a_{i j} \partial_{i} C_{j k}=-a_{i k} y_{j} \partial_{i} \partial_{k}+a_{j k} y_{i} \partial_{j} \partial_{k}+a_{i j} y_{k} \partial_{i} \partial_{j} \\
{\underset{C_{i j}, C_{j k}, C_{k l}}{\longrightarrow}}^{*} y_{i}\left(-F_{j k}\right)+y_{k}\left(-F_{i j}\right)-y_{j}\left(-F_{i k}\right)=0 .
\end{gathered}
$$

The $S$-pair of $C_{i j}$ and $C_{i k}$ and that of $C_{i k}$ and $C_{j k}$ are also reduced to 0 .

$S$-pair of $D_{i}$ and $A_{j}$. The initial monomials $\operatorname{in}_{<}\left(D_{i}\right)=\operatorname{in}_{<}\left(\partial_{i}\right)^{3}, \mathrm{in}_{<}\left(A_{j}\right)=$ $\mathrm{in}_{<}\left(\partial_{j j}\right)$ are coprime. When $i<j$, operators $D_{i}$ and $A_{j}$ commute. By Lemma 1. we have

$$
S\left(D_{i}, A_{j}\right){\overrightarrow{D_{i}, A_{j}}}^{*} 0 .
$$

When $i>j$, by Lemmas 1 and 3 , we have

$$
S\left(D_{i}, A_{j}\right){\underset{D_{i}, A_{j}}{\longrightarrow}}^{*}\left[D_{i}, A_{j}\right]=2 a_{j i}^{-2} \partial_{j} C_{j i}{C_{j i}}^{*} 0 .
$$


When $i=j$, by Lemmas 1 and 3 , we have

$$
S\left(D_{i}, A_{i}\right) \underset{D_{i}, A_{j}}{\longrightarrow}\left[D_{i}, A_{i}\right]=\sum_{l=1}^{i-1} 2 a_{l i}^{-2} \partial_{l} C_{l i}{\underset{C_{1 i}, \ldots, C_{i-1 i}}{\longrightarrow}}^{*} 0 .
$$

$S$-pair of $D_{i}$ and $B$. When $i=1$, the $S$-pair is $S\left(D_{1}, B\right)=\partial_{1} B-\partial_{1} B=0$.

When $i>1$, the initial monomials $\operatorname{in}_{<}\left(D_{i}\right)=\operatorname{in}_{<}\left(\partial_{i}\right)^{3}, \operatorname{in}_{<}(B)=\operatorname{in}_{<}\left(\partial_{1}\right)^{2}$ are coprime. By Lemmas 1 and 3 , we have

$$
S\left(D_{i}, B\right) \underset{D_{i}, B}{\longrightarrow}\left[D_{i}, B\right]=0 .
$$

$S$-pair of $D_{i}$ and $D_{j}$. The initial monomials $\operatorname{in}_{<}\left(D_{i}\right)=\operatorname{in}_{<}\left(\partial_{i}\right)^{3}, \operatorname{in}_{<}\left(D_{j}\right)=$ $\operatorname{in}_{<}\left(\partial_{j}\right)^{3}$ are coprime. By Lemmas 1 and 3 we have

$$
S\left(D_{i}, D_{j}\right) \underset{D_{i}, D_{j}}{\longrightarrow}\left[D_{i}, D_{j}\right] \underset{B, C_{1 j}, \ldots, C_{i j}}{\longrightarrow} * \text {. }
$$

$S$-pair of $C_{i j}$ and $D_{k}$. The initial monomials are $\operatorname{in}_{<}\left(C_{i j}\right)=\mathrm{in}_{<}\left(\partial_{i}\right) \operatorname{in}_{<}\left(\partial_{j}\right)$, $\operatorname{in}_{<}\left(D_{k}\right)=\operatorname{in}_{<}\left(\partial_{k}\right)^{3}$. When $i \neq k$ and $j \neq k$, the initial monomials are coprime. By Lemmas 1 and 3, we have

$$
S\left(C_{i j}, D_{k}\right){\underset{C_{i j}, D_{k}}{\longrightarrow}}^{*}\left[C_{i j}, D_{k}\right]{\underset{C_{i k}, C_{j k}}{\longrightarrow}}^{*} 0 .
$$

When $i=k$, the initial monomials $\operatorname{in}_{<}\left(C_{i j}\right)=\operatorname{in}_{<}\left(\partial_{i}\right) \operatorname{in}_{<}\left(\partial_{j}\right), \operatorname{in}_{<}\left(D_{i}\right)=$ $\operatorname{in}_{<}\left(\partial_{i}\right)^{3}$ are not coprime. This case needs a care of an order of applying reductions. We will reduce the $S$-pair by $D_{j}$ and then reduce remainders by $C_{i j}$ 's.

$$
\begin{aligned}
& S\left(C_{i j}, D_{i}\right)=\partial_{i}^{2} C_{i j}-a_{i j} \partial_{j} D_{i} \\
& =\partial_{i}^{2} F_{i j}-a_{i j} \partial_{i} \partial_{j}^{3}-a_{i j} \partial_{j}\left(\sum_{l=i+1, l \neq j}^{n+1} \partial_{i} \partial_{l}^{2}-\partial_{i} r^{2}-\sum_{l=1}^{i-1} \partial_{l} a_{l i}^{-1} F_{l i}\right) \\
& {\overrightarrow{D_{j}}}^{*} \partial_{i}^{2} F_{i j}+a_{i j} \partial_{i}\left(\sum_{l=j+1}^{n+1} \partial_{j} \partial_{l}^{2}-\partial_{j} r^{2}-\sum_{l=1}^{j-1} \partial_{l} a_{l j}^{-1} F_{l j}\right) \\
& -a_{i j} \partial_{j}\left(\sum_{l=i+1, l \neq j}^{n+1} \partial_{i} \partial_{l}^{2}-\partial_{i} r^{2}-\sum_{l=1}^{i-1} \partial_{l} a_{l i}^{-1} F_{l i}\right) \\
& =-a_{i j} \partial_{i}\left(\partial_{i+1} a_{i+1 j}^{-1} C_{i+1 j}+\cdots+\partial_{j-1} a_{j-1 j}^{-1} C_{j-1 j}\right) \\
& -a_{i j} \partial_{i}\left(\sum_{l=1}^{i-1} a_{l j}^{-1} F_{l j}\right)+a_{i j} \partial_{j}\left(\sum_{l=1}^{i-1} \partial_{l} a_{l i}^{-1} F_{l i}\right) \\
& {\underset{C_{i+1 j}, \ldots, C_{j-1 j}}{\longrightarrow}}^{*}-a_{i j} \partial_{i}\left(\sum_{l=1}^{i-1} a_{l j}^{-1} F_{l j}\right)+a_{i j} \partial_{j}\left(\sum_{l=1}^{i-1} \partial_{l} a_{l i}^{-1} F_{l i}\right) \\
& =-a_{i j} \sum_{l=1}^{i-1}\left(a_{l j}^{-1} \partial_{l} \partial_{i} F_{l j}-a_{l i}^{-1} \partial_{l} \partial_{j} F_{l i}\right) \text {. }
\end{aligned}
$$


Since $a_{l j}^{-1} \partial_{l} \partial_{i} F_{l j}-a_{l i}^{-1} \partial_{l} \partial_{j} F_{l i} \underset{C_{l j}, C_{l i}, C_{i j}}{\longrightarrow} 0$, the $S$-pair $S\left(C_{i j}, D_{i}\right)$ is reduced to 0 .

When $j=k$, the initial monomials $\operatorname{in}_{<}\left(C_{i j}\right)=\operatorname{in}_{<}\left(\partial_{i}\right) \operatorname{in}_{<}\left(\partial_{j}\right), \operatorname{in}_{<}\left(D_{j}\right)=$ $\operatorname{in}_{<}\left(\partial_{j}\right)^{3}$ are not coprime. This case also needs a care of an order of applying reductions.

$$
\begin{aligned}
S\left(C_{i j}, D_{j}\right) & =\partial_{j}^{2} C_{i j}-a_{i j} \partial_{i} D_{j} \\
& =F_{i j}\left(\partial_{i}^{2}+\partial_{j}^{2}\right)-a_{i j} \partial_{i}\left(\sum_{l=j+1}^{n+1} \partial_{j} \partial_{l}^{2}-\partial_{j} r^{2}-\sum_{l=1, l \neq i}^{j-1} \partial_{l} a_{l j}^{-1} F_{l j}\right) \\
& \vec{B}^{*} F_{i j}\left(-\sum_{l=1, l \neq i, j}^{n+1} \partial_{l}^{2}+r^{2}\right)-a_{i j} \partial_{i}\left(\sum_{l=j+1}^{n+1} \partial_{j} \partial_{l}^{2}-\partial_{j} r^{2}-\sum_{l=1, l \neq i}^{j-1} \partial_{l} a_{l j}^{-1} F_{l j}\right) \\
& =F_{i j}\left(-\sum_{l=1, l \neq i, j}^{n+1} \partial_{l}^{2}\right)+r^{2}\left(a_{i j} \partial_{i} \partial_{j}+F_{i j}\right)-a_{i j} \partial_{i}\left(\sum_{l=j+1}^{n+1} \partial_{j} \partial_{l}^{2}-\sum_{l=1, l \neq i}^{j-1} \partial_{l} a_{l j}^{-1} F_{l j}\right) \\
& C_{i j}{ }^{*} F_{i j}\left(-\sum_{l=1, l \neq i, j}^{n+1} \partial_{l}^{2}\right)-a_{i j} \partial_{i}\left(\sum_{l=j+1}^{n+1} \partial_{j} \partial_{l}^{2}-\sum_{l=1, l \neq i}^{j-1} \partial_{l} a_{l j}^{-1} F_{l j}\right) \\
& =\left(\sum_{l=j+1}^{n+1} \partial_{l}^{2}\right)\left(-a_{i j} \partial_{i} \partial_{j}-F_{i j}\right)-F_{i j} \sum_{l=1, l \neq i}^{j-1} \partial_{l}^{2}+a_{i j} \partial_{i} \sum_{l=1, l \neq i}^{j-1} \partial_{l} a_{l j}^{-1} F_{l j} \\
& \underset{C_{i j}}{\longrightarrow}-F_{i j} \sum_{l=1, l \neq i}^{j-1} \partial_{l}^{2}+a_{i j} \partial_{i} \sum_{l=1, l \neq i}^{j-1} \partial_{l} a_{l j}^{-1} F_{l j} \\
& =\sum_{l=1, l \neq i}^{j-1} \partial_{l} a_{i j}\left(-a_{i j}^{-1} \partial_{l} F_{i j}+a_{l j}^{-1} \partial_{i} F_{l j}\right) .
\end{aligned}
$$

Since $-a_{i j}^{-1} \partial_{l} F_{i j}+a_{l j}^{-1} \partial_{i} F_{l j} \underset{C_{l j}, C_{l i}, C_{i j}}{\longrightarrow}$, the $S$-pair $S\left(C_{i j}, D_{j}\right)$ is reduced to 0 .

$S$-pair of $E$ and $A_{i}$. The initial monomials $\operatorname{in}_{<}(E)=\operatorname{in}_{<}\left(\partial_{r}\right)$, in $_{<}\left(A_{i}\right)=$ $\mathrm{in}_{<}\left(\partial_{i i}\right)$ are coprime. By Lemmas 1 and 4 , we have

$$
S\left(A_{i}, E\right) \underset{A_{i}, E}{\longrightarrow}\left[A_{i}, E\right]=0 .
$$

$S$-pair of $E$ and $B$. The initial monomials $\operatorname{in}_{<}(E)=\operatorname{in}_{<}\left(\partial_{r}\right), \mathrm{in}_{<}(B)=$ in $_{<}\left(\partial_{1}\right)^{2}$ are coprime. By Lemmas 1 and 4 , we have

$$
S(B, E) \overrightarrow{B, E}^{*}[B, E]=-2 B \vec{B}^{*} 0 .
$$

$S$-pair of $E$ and $C_{i j}$. The initial monomials $\operatorname{in}_{<}(E)=\operatorname{in}_{<}\left(\partial_{r}\right), \mathrm{in}_{<}\left(C_{i j}\right)=$ $\mathrm{in}_{<}\left(\partial_{i}\right) \mathrm{in}_{<}\left(\partial_{j}\right)$ are coprime. By Lemmas 1 and 4 , we have

$$
S\left(C_{i j}, E\right) \underset{C_{i j}, E}{\longrightarrow}\left[C_{i j}, E\right]=0
$$


$S$-pair of $D_{i}$ and $E$. The initial monomials $\operatorname{in}_{<}(E)=\operatorname{in}_{<}\left(\partial_{r}\right), \operatorname{in}_{<}\left(D_{i}\right)=$ $\mathrm{in}_{<}\left(\partial_{i}\right)^{3}$ are coprime. By Lemmas 1 and 4 , we have

$$
S\left(D_{i}, E\right) \underset{D_{i}, E}{\longrightarrow}\left[D_{i}, E\right]=-3 D_{i}-2 \sum_{k=1}^{i-1} a_{k i}^{-1} \partial_{k} C_{k i}{\underset{D_{i}, C_{1 i}, \ldots, C_{i-1 i}}{\longrightarrow}}^{*} 0 .
$$

We have proved that any $S$-pair is reduced to 0. By Buchberger's criterion, the set $\left\{A_{i}, B, C_{i j}, D_{k}, E\right\}$ is a Gröbner basis of $R \tilde{I}$. Q.E.D.

\section{Gröbner Deformation of the Fisher-Bingham System}

Consider the system of differential equations $I \cdot f=0$. Intuitively speaking, we want to prove that the system $I$ can be deformed to the diagonal system $\tilde{I}$ without increasing the holonomic rank. This can be done by a Gröbner basis computation with a weight vector $(-w, w)$ [6, Theorem 2.2.1]. However a straightforward calculation does not seem to be easy. We need to use some technical tricks to determine a suitable Gröbner deformation. Since these tricks may look too technical for the general $n$, we explain them in the case of $n=1$ in Section 4 to clarify our idea without technical details of this section. Readers are expected to refer to the Section 4 when technicalities get complicated.

We will introduce new indeterminates to make our Gröbner basis computation possible by hand with employing the idea of the proof of [6, Theorem 3.1.3]. Let $a_{p q}, b_{i}, c_{i}, d(1 \leq p \leq q \leq n+1,1 \leq i \leq n+1)$ be constants, which we call slack variables when they are regarded as indeterminates. We put $g=\left(r^{d^{3}} \prod_{p \leq q} x_{p q}^{a_{p q}^{3}}\right) f$ and make a change of the independent variables $y_{i}$ by $y_{i}+b_{i} c_{i}$. Then, the system of differential equations for the function $g$ is $I^{\prime} \cdot g=0$ where $I^{\prime}$ is the left ideal in $D$ generated by the set of operators $G^{\prime}=\left\{A_{p q}^{\prime}, B, C_{i j}^{\prime}, E^{\prime}\right\}$ where

$$
\begin{aligned}
& A_{p q}^{\prime}=x_{p q} \partial_{p q}-x_{p q} \partial_{p} \partial_{q}-a_{p q}^{3}, \\
& \begin{aligned}
B= & \sum_{i=1}^{n+1} \partial_{i}^{2}-r^{2}
\end{aligned} \\
& \begin{aligned}
C_{i j}^{\prime}= & x_{i j} \partial_{i}^{2}+2\left(x_{j j}-x_{i i}\right) \partial_{i} \partial_{j}-x_{i j} \partial_{j}^{2} \\
& \quad+\sum_{s \neq i, j}\left(x_{s j} \partial_{i} \partial_{s}-x_{i s} \partial_{j} \partial_{s}\right)+\left(y_{j}+b_{j} c_{j}\right) \partial_{i}-\left(y_{i}+b_{i} c_{i}\right) \partial_{j},
\end{aligned} \\
& \quad E^{\prime}=r \partial_{r}-2 \sum_{i \leq j} x_{i j} \partial_{i} \partial_{j}-\sum_{i=1}^{n+1}\left(y_{i}+b_{i} c_{i}\right) \partial_{i}-n-d^{3} .
\end{aligned}
$$

The key fact is that the holonomic rank of $I$ for $f$ agrees with the holonomic rank of $I^{\prime}$ for $g$ for any constants $a_{p q}, b_{i}, c_{i}, d$. 
Let us make the same change of the variables for the diagonal system; let $\tilde{I}^{\prime}$ be the left ideal of $D$ generated by the set of operators $\tilde{G}^{\prime}=\left\{\tilde{A}_{i i}^{\prime}, B, \tilde{C}_{i j}^{\prime}, \tilde{E}^{\prime}, x_{i j} \partial_{i j}-\right.$ $\left.a_{i j}^{3}(i \neq j)\right\}$ where

$$
\begin{aligned}
& \tilde{A}_{i i}^{\prime}=x_{i i} \partial_{i}^{2}-x_{i i} \partial_{i i}-a_{i i}^{3} \quad(1 \leq i \leq n+1), \\
& B=\sum_{i=1}^{n+1} \partial_{i}^{2}-r^{2}, \\
& \tilde{C}_{i j}^{\prime}=2\left(x_{j j}-x_{i i}\right) \partial_{i} \partial_{j}+\left(y_{j}+b_{j} c_{j}\right) \partial_{i}-\left(y_{i}+b_{i} c_{i}\right) \partial_{j} \quad(1 \leq i<j \leq n+1), \\
& \tilde{E}^{\prime}=r \partial_{r}-2 \sum_{i=1}^{n+1} x_{i i} \partial_{i}^{2}-\sum_{i=1}^{n+1}\left(y_{i}+b_{i} c_{i}\right) \partial_{i}-n-d^{3} .
\end{aligned}
$$

The holonomic ranks of $\tilde{I}$ and $\tilde{I}^{\prime}$ agree.

Define the weight vector $w$ by $w_{i j}=1,(i \neq j), w_{i i}=0, w_{k}=0$, and $w_{r}=0$. Here, $w_{i j}$ stands for $\partial_{i j}, w_{k}$ stands for $\partial_{k}$, and $w_{r}$ stands for $\partial_{r}$. The initial form in $\operatorname{in}_{(-w, w)}(\ell), \ell \in D$, is the sum of the highest $(-w, w)$-degree terms in $\ell$ and $\operatorname{in}_{(-w, w)}\left(I^{\prime}\right)$ is the left ideal generated by $\ell \in I^{\prime}$ where the weight $-w$ stands for space variables $x_{i j}, y_{k}, r$ corresponding to differential operators $\partial_{i j}, \partial_{k}, \partial_{r}$ respectively (see, e.g., [6, Chapter 1]).

Theorem 3 For generic complex numbers $a_{p q}, b_{i}, c_{i}, d$, we have

$$
\operatorname{in}_{(-w, w)}\left(I^{\prime}\right)=\tilde{I}^{\prime} .
$$

In order to prove the theorem, we regard $a_{p q}, b_{i}, c_{i}, d$ as ring variables with the weight 0 and consider the following homogenized system of $I^{\prime}$ :

$$
\begin{aligned}
& A_{p q}^{\prime h}=h x_{p q} \partial_{p q}-x_{p q} \partial_{p} \partial_{q}-a_{p q}^{3}, \\
& B=\sum_{i=1}^{n+1} \partial_{i}^{2}-r^{2}, \\
& C_{i j}^{\prime h}=x_{i j} \partial_{i}^{2}+2\left(x_{j j}-x_{i i}\right) \partial_{i} \partial_{j}-x_{i j} \partial_{j}^{2} \\
& +\sum_{s \neq i, j}\left(x_{s j} \partial_{i} \partial_{s}-x_{i s} \partial_{j} \partial_{s}\right)+\left(h y_{j}+b_{j} c_{j}\right) \partial_{i}-\left(h y_{i}+b_{i} c_{i}\right) \partial_{j}, \\
& E^{\prime h}=h r \partial_{r}-2 \sum_{i \leq j} x_{i j} \partial_{i} \partial_{j}-\sum_{i=1}^{n+1}\left(h y_{i}+b_{i} c_{i}\right) \partial_{i}-n h^{3}-d^{3} .
\end{aligned}
$$

Let $I^{\prime h}$ be a left $D^{h}[a, b, c, d]$-ideal generated by the set of operators $G^{\prime h}:=$ $\left\{A_{p q}^{\prime h}, B, C_{i j}^{\prime h}, E^{\prime h}\right\}$, where $D^{h}[a, b, c, d]$ is the homogenized Weyl algebra of the ring $D[a, b, c, d]=\mathbf{C}[a, b, c, d]\left\langle x_{i j}, y_{k}, r, \partial_{i j}, \partial_{k}, \partial_{r}\right\rangle$ with the homogenization variable $h$ (see, e.g., [5, Section 9]). We introduce a new term order $<_{(-w, w, 0)}^{h}$ over $D^{h}[a, b, c, d]$, which compares the total degree first, $(-w, w, 0)$-degree second, otherwise we apply the following block order as a tie breaker: $d \gg r \gg\left\{a_{p q} \mid\right.$ $i \leq j\} \gg\left\{b_{k}\right\} \gg\left\{c_{k}\right\} \gg\left\{y_{k}\right\} \gg \partial_{r} \gg\left\{\partial_{i j} \mid i<j\right\} \gg\left\{\partial_{i i}\right\} \gg\left\{\partial_{k}\right\} \gg\left\{x_{i j} \mid\right.$ 
$i<j\} \gg\left\{x_{i i}\right\} \gg h$. Here, the block $\left\{b_{k}\right\}$ has a lexicographic order so that $b_{1}>b_{2}>\cdots>b_{n+1}$. The use of this tie breaker is a key of our calculation. Although, the initial monomial $\operatorname{in}_{<_{(-w, w, 0)}^{h}}(\ell)$ is an element of the associated commutative ring, we denote it by the associated element in $D^{h}[a, b, c, d]$ as long as no confusion arises. For example, we denote $\operatorname{in}_{<_{(-w, w, 0)}^{h}}\left(\partial_{i j}\right)$ by $\partial_{i j}$ instead of $\xi_{i j}$.

Proposition $2 A$ Gröbner basis of $I^{\text {h }}$ with respect to $<_{(-w, w, 0)}^{h}$ is $G^{\prime h}=$ $\left\{A_{p q}^{\prime h}, B, C_{i j}^{\prime h}, E^{\prime h}\right\}$.

We need four lemmas for proving the proposition. These can be obtained by a straightforward calculation.

Lemma 5 The initial monomials of the generators of $I^{\text {th }}$ are

1. $\operatorname{in}_{<_{(-w, w, 0)}^{h}}\left(A_{p q}^{\prime h}\right)=-a_{p q}^{3}$,

2. $\operatorname{in}_{<{ }_{(-w, w, 0)}^{h}}(B)=-r^{2}$,

3. $\operatorname{in}_{<(-w, w, 0)}^{h}\left(C_{i j}^{\prime h}\right)=-b_{i} c_{i} \partial_{j}$,

4. $\operatorname{in}_{<{ }_{(-w, w 0)}^{h}}\left(E^{\prime h}\right)=-d^{3}$.

In particular, they are pairwise coprime expect in the case that the pair $\operatorname{in}_{<_{(-w, w, 0)}^{h}}\left(C_{i j}^{\prime h}\right)$ and $\operatorname{in}_{<_{(-w, w, 0)}^{h}}\left(C_{i k}^{\prime h}\right)$ and the pair $\operatorname{in}_{<_{(-w, w, 0)}^{h}}\left(C_{i k}^{\prime h}\right)$ and $\operatorname{in}_{<_{(-w, w, 0)}^{h}}\left(C_{j k}^{\prime h}\right)$.

Lemma 6 The commutators of two generators of $I^{\text {th }}$ are

1. $\left[A_{p q}^{\prime h}, A_{r s}^{\prime h}\right]=\left[A_{p q}^{\prime h}, B\right]=\left[A_{p q}^{\prime h}, C_{i j}^{\prime h}\right]=\left[A_{p q}^{\prime h}, E^{\prime h}\right]=0 \quad$ (for any $\left.p, q, r, s, i, j\right)$,

2. $\left[B, C_{i j}^{\prime h}\right]=0,\left[B, E^{\prime h}\right]=-2 h B$,

3. $\left[C_{i j}^{\prime h}, C_{k l}^{\prime h}\right]=0 \quad(\{i, j\} \cap\{k, l\}=\emptyset)$,

4. $\left[C_{i j}^{\prime h}, C_{j k}^{\prime h}\right]=C_{k i}^{\prime h}:=-C_{i k}^{\prime h},\left[C_{i j}^{\prime h}, C_{i k}^{\prime h}\right]=C_{j k}^{\prime h},\left[C_{i k}^{\prime h}, C_{j k}^{\prime h}\right]=C_{i j}^{\prime h} \quad(i<j<k)$,

5. $\left[C_{i j}^{\prime h}, E^{\prime h}\right]=0$.

Lemma 7 The following holds for $S$-pairs of generators of $I^{\text {th }}$ :

1. $S\left(A_{p q}^{\prime h}, A_{r s}^{\prime h}\right), S\left(A_{p q}^{\prime h}, B\right), S\left(A_{p q}^{\prime h}, C_{i j}^{\prime h}\right), S\left(A_{p q}^{\prime h}, E^{\prime h}\right) \longrightarrow^{*} 0 \quad$ (for any $\left.p, q, r, s, i, j\right)$,

2. $S\left(B, C_{i j}^{\prime h}\right) \longrightarrow^{*} 0, S\left(B, E^{\prime h}\right) \longrightarrow^{*} 0$,

3. $S\left(C_{i j}^{\prime h}, C_{k l}^{\prime h}\right) \longrightarrow{ }^{*} 0 \quad(\{i, j\} \cap\{k, l\}=\emptyset)$,

4. $S\left(C_{i j}^{\prime h}, C_{j k}^{\prime h}\right) \longrightarrow^{*} 0 \quad(i<j<k)$,

5. $S\left(C_{i j}^{\prime h}, E^{\prime h}\right) \longrightarrow^{*} 0$.

Proof. Lemma 1 holds in the homogenized Weyl algebra $D^{h}[a, b, c, d]$ too. Therefore, these follow from Lemmas 5 and 6. Q.E.D. 
Lemma 8 We put $\hat{C}_{i j}^{\prime h}:=b_{j} c_{j} \partial_{i}-b_{i} c_{i} \partial_{j}$ and $\check{C}_{i j}^{\prime h}:=C_{i j}^{\prime h}-\hat{C}_{i j}^{\prime h}$. Then the following cyclic relations hold:

1. $\partial_{k} \hat{C}_{i j}^{\prime h}+\partial_{i} \hat{C}_{j k}^{\prime h}+\partial_{j} \hat{C}_{k i}^{\prime h}=\hat{C}_{i j}^{\prime h} \partial_{k}+\hat{C}_{j k}^{\prime h} \partial_{i}+\hat{C}_{k i}^{\prime h} \partial_{j}=0$,

2. $\partial_{k} \check{C}_{i j}^{\prime h}+\partial_{i} \check{C}_{j k}^{\prime h}+\partial_{j} \check{C}_{k i}^{\prime h}=\check{C}_{i j}^{\prime h} \partial_{k}+\check{C}_{j k}^{\prime h} \partial_{i}+\check{C}_{k i}^{\prime h} \partial_{j}=0$,

3. $\partial_{k} C_{i j}^{\prime h}+\partial_{i} C_{j k}^{\prime h}+\partial_{j} C_{k i}^{\prime h}=C_{i j}^{\prime h} \partial_{k}+C_{j k}^{\prime h} \partial_{i}+C_{k i}^{\prime h} \partial_{j}=0$,

4. $b_{k} c_{k} \hat{C}_{i j}^{\prime h}+b_{i} c_{i} \hat{C}_{j k}^{\prime h}+b_{j} c_{j} \hat{C}_{k i}^{\prime h}=\hat{C}_{i j}^{\prime h} b_{k} c_{k}+\hat{C}_{j k}^{\prime h} b_{i} c_{i}+\hat{C}_{k i}^{\prime h} b_{j} c_{j}=0$.

Proof of Proposition [2, By Lemma 7, we only need to check that the $S$-pairs $S\left(C_{i j}^{\prime h}, C_{i k}^{\prime h}\right)$ and $S\left(C_{i k}^{\prime h}, C_{j k}^{\prime h}\right)$ are reduced to zero.

The former is $S\left(C_{i j}^{\prime h}, C_{i k}^{\prime h}\right)=\partial_{k} C_{i j}^{\prime h}-\partial_{j} C_{i k}^{\prime h}=\partial_{k} C_{i j}^{\prime h}+\partial_{j} C_{k i}^{\prime h}$, because the initial monomials are $\operatorname{in}_{<_{(-w, w, 0)}^{h}}\left(C_{i j}^{\prime h}\right)=-b_{i} c_{i} \partial_{j}$ and $\operatorname{in}_{<_{(-w, w, 0)}^{h}}\left(C_{i k}^{\prime h}\right)=-b_{i} c_{i} \partial_{k}$. The $S$-pair is equal to $-\partial_{i} C_{j k}^{\prime h}$ by the 3 rd formula of Lemma 8 This implies that it is reduced to zero.

The latter is $S\left(C_{i k}^{\prime h}, C_{j k}^{\prime h}\right)=b_{j} c_{j} C_{i k}^{\prime h}-b_{i} c_{i} C_{j k}^{\prime h}$, because the initial monomials are $\operatorname{in}_{<_{(-w, w, 0)}^{h}}\left(C_{i k}^{\prime h}\right)=-b_{i} c_{i} \partial_{k}$ and $\operatorname{in}_{<_{(-w, w, 0)}^{h}}\left(C_{j k}^{\prime h}\right)=-b_{j} c_{j} \partial_{k}$.

Firstly, we show that it can be expressed as

$$
\begin{aligned}
S\left(C_{i k}^{\prime h}, C_{j k}^{\prime h}\right) & =\left(\sum_{s=1}^{n+1}\left(\delta_{k s}+1\right) x_{k s} \partial_{s}+h y_{k}+b_{k} c_{k}\right) C_{i j}^{\prime h} \\
& +\left(\sum_{s=1}^{n+1}\left(\delta_{i s}+1\right) x_{i s} \partial_{s}+h y_{i}\right) C_{j k}^{\prime h} \\
& +\left(\sum_{s=1}^{n+1}\left(\delta_{j s}+1\right) x_{j s} \partial_{s}+h y_{j}\right) C_{k i}^{\prime h}
\end{aligned}
$$

Here, the symbol $\delta$ is the Kronecker delta. This expression of the $S$-pair is a key of our proof. Since each monomial of the left hand side (LHS in short) has at least one variable in $b_{i}, b_{j}$ or $b_{k}$, we divide the right hand side (RHS in short) into two parts: $S_{1}$ contains these variables, $S_{2}$ does not contain them. Then,

$$
\begin{aligned}
S_{2} & =\left(\sum_{s=1}^{n+1}\left(\delta_{k s}+1\right) x_{k s} \partial_{s}+h y_{k}\right) \check{C}_{i j}^{\prime h}+\left(\sum_{s=1}^{n+1}\left(\delta_{i s}+1\right) x_{i s} \partial_{s}+h y_{i}\right) \check{C}_{j k}^{\prime h} \\
& +\left(\sum_{s=1}^{n+1}\left(\delta_{j s}+1\right) x_{j s} \partial_{s}+h y_{j}\right) \check{C}_{k i}^{\prime h}
\end{aligned}
$$


is equal to zero by a straightforward calculation and we have

$$
\begin{aligned}
S_{1}= & b_{k} c_{k} C_{i j}^{\prime h}+\left(\sum_{s=1}^{n+1}\left(\delta_{k s}+1\right) x_{k s} \partial_{s}+h y_{k}\right) \hat{C}_{i j}^{\prime h} \\
& \quad+\left(\sum_{s=1}^{n+1}\left(\delta_{i s}+1\right) x_{i s} \partial_{s}+h y_{i}\right) \hat{C}_{j k}^{\prime h}+\left(\sum_{s=1}^{n+1}\left(\delta_{j s}+1\right) x_{j s} \partial_{s}+h y_{j}\right) \hat{C}_{k i}^{\prime h} \\
= & b_{k} c_{k} C_{i j}^{\prime h} \\
+ & b_{j} c_{j}\left(\sum_{s=1}^{n+1}\left(\delta_{k s}+1\right) x_{k s} \partial_{s} \partial_{i}+h y_{k} \partial_{i}\right)-b_{i} c_{i}\left(\sum_{s=1}^{n+1}\left(\delta_{k s}+1\right) x_{k s} \partial_{s} \partial_{j}+h y_{k} \partial_{j}\right) \\
& +b_{k} c_{k}\left(\sum_{s=1}^{n+1}\left(\delta_{i s}+1\right) x_{i s} \partial_{s} \partial_{j}+h y_{i} \partial_{j}\right)-b_{j} c_{j}\left(\sum_{s=1}^{n+1}\left(\delta_{i s}+1\right) x_{i s} \partial_{s} \partial_{k}+h y_{i} \partial_{k}\right) \\
+ & b_{i} c_{i}\left(\sum_{s=1}^{n+1}\left(\delta_{j s}+1\right) x_{j s} \partial_{s} \partial_{k}+h y_{j} \partial_{k}\right)-b_{k} c_{k}\left(\sum_{s=1}^{n+1}\left(\delta_{j s}+1\right) x_{j s} \partial_{s} \partial_{i}+h y_{j} \partial_{i}\right) \\
= & b_{j} c_{j} C_{i k}^{\prime h}-b_{i} c_{i} C_{j k}^{\prime h}=S\left(C_{i k}^{\prime h}, C_{j k}^{\prime h}\right) .
\end{aligned}
$$

Finally, we show that the expression (3) is a standard representation. We note that the RHS of (3) has three terms, which appear in the first, in the second and in the third lines of (3) respectively. We may show that the initial monomial of the LHS is no less than the initial monomials of the three terms of the RHS with respect to the term order $<_{(-w, w, 0)}^{h}$. The initial monomial of the LHS $b_{i} c_{i} b_{k} c_{k} \partial_{j}$ coincides with that of the 1 st term of the RHS. Moreover, all monomials appearing in the 2nd and the 3rd term of the RHS have the same total degree 5 and $(-w, w, 0)$-degree 0 , and they have a degree at most one with respect to $b_{i}, b_{j}$ and $b_{k}$. This implies that they are less than $b_{i} c_{i} b_{k} c_{k} \partial_{j}$. Thus, we have shown that the expression is a standard representation and the $S$-pair is reduced to zero.

Since all $S$-pairs are reduced to zero, the conclusion follows from Buchberger's criterion. Q.E.D.

Proof of Theorem [3. Let $I^{\prime}(a, b, c, d)$ be the left ideal generated by $G^{\prime}$ in the ring $D[a, b, c, d]$. It follows from Proposition 2 and [6, Theorem 1.2.4] that $\left.G^{\prime h}\right|_{h=1}=$ $G^{\prime}$ is a Gröbner basis of $I^{\prime}(a, b, c, d)$ with respect to $<_{(-w, w, 0)}$. Therefore, we have

$$
\operatorname{in}_{<_{(-w, w, 0)}}\left(I^{\prime}(a, b, c, d)\right)=\left\langle\operatorname{in}_{<_{(-w, w, 0)}}\left(G^{\prime}\right)\right\rangle=\left\langle\tilde{G}^{\prime}\right\rangle \quad \text { in } D[a, b, c, d] .
$$

We may replace $D[a, b, c, d]$ by $D(a, b, c, d)=\mathbf{C}(a, b, c, d)\left\langle x_{i j}, y_{k}, r, \partial_{i j}, \partial_{k}, \partial_{r}\right\rangle$. Here, $\mathbf{C}(a, b, c, d)$ is the rational function field with variables $a=\left(a_{p q}\right), b=$ $\left(b_{i}\right), c=\left(c_{i}\right)$ and $d$. Then, the following holds:

$$
\operatorname{in}_{(-w, w)}\left(I^{\prime}(a, b, c, d)\right)=\left\langle\tilde{G}^{\prime}\right\rangle \quad \text { in } D(a, b, c, d) .
$$

This implies the conclusion. Q.E.D. 
Proof of the Main theorem[1. It follows from (2) that $\operatorname{rank}(I) \geq \operatorname{rank}\left(\operatorname{in}_{(-w, w)}\left(I^{\prime}\right)\right)$ by Theorem 2.2.1 in [6]. Therefore, we have $\operatorname{rank}(I) \geq 2 n+2$ by Theorem 2 , The opposite inequality follows from Theorem 3 of [4]. Q.E.D.

\section{A Proof in the case of $n=1$}

In order to clarify ideas of the proof in Section 3, we present a proof of our theorem in the case of $n=1$.

The 1-dimensional Fisher-Bingham system of differential equations $I \subset$ $\mathbf{C}\left\langle x_{11}, x_{12}, x_{22}, y_{1}, y_{2}, r, \partial_{11}, \partial_{12}, \partial_{22}, \partial_{1}, \partial_{2}, \partial_{r}\right\rangle$ is

$$
\begin{aligned}
I= & \left\langle A_{11}=\partial_{11}-\partial_{1}^{2}, A_{12}=\partial_{12}-\partial_{1} \partial_{2},\right. \\
& A_{22}=\partial_{22}-\partial_{2}^{2}, \\
& B=\partial_{1}^{2}+\partial_{2}^{2}-r^{2}, \\
& C_{12}=x_{12} \partial_{1}^{2}+2\left(x_{22}-x_{11}\right) \partial_{1} \partial_{2}-x_{12} \partial_{2}^{2}+y_{2} \partial_{1}-y_{1} \partial_{2}, \\
& \left.E=r \partial_{r}-2\left(x_{11} \partial_{1}^{2}+x_{12} \partial_{1} \partial_{2}+x_{22} \partial_{2}^{2}\right)-\left(y_{1} \partial_{1}+y_{2} \partial_{2}\right)-1\right\rangle .
\end{aligned}
$$

The upper bound of $\operatorname{rank}(I)$ is $2 \cdot 1+2=4$ as given in [4, Theorem 3]. We will show the lower bound of the $\operatorname{rank}(I)$ is 4 by using the following general inequality [6, Theorem 2.2.1]:

$$
\operatorname{rank}(I) \geq \operatorname{in}_{(-w, w)}(I) .
$$

Firstly, we make some change of variables, because it seems to be difficult to calculate $\operatorname{in}_{(-w, w)}(I)$ directly. Let $a_{11}, a_{12}, a_{22}, b_{1}, b_{2}, c_{1}, c_{2}, d$ be constants, which will be used as slack variables. We put $g=r^{d^{3}} x_{11}^{a_{11}^{3}} x_{12}^{a_{12}^{3}} x_{22}^{a_{22}^{3}} f$ where the function $f$ is a solution of the system of differential equations $I \cdot f=0$. Moreover, we make a change of variables $y_{1}, y_{2}$ by $y_{1}+b_{1} c_{1}, y_{2}+b_{2} c_{2}$ respectively. Then, the system of differential equations for $g$ is given by $I^{\prime} \cdot g=0$, where

$$
\begin{aligned}
& I^{\prime}=\left\langle A_{11}^{\prime}=x_{11} \partial_{11}-x_{11} \partial_{1}^{2}-a_{11}^{3}, A_{12}^{\prime}=x_{12} \partial_{12}-x_{12} \partial_{1} \partial_{2}-a_{12}^{3},\right. \\
& A_{22}^{\prime}=x_{22} \partial_{22}-x_{22} \partial_{2}^{2}-a_{22}^{3}, \\
& B=\partial_{1}^{2}+\partial_{2}^{2}-r^{2}, \\
& C_{12}^{\prime}=x_{12} \partial_{1}^{2}+2\left(x_{22}-x_{11}\right) \partial_{1} \partial_{2}-x_{12} \partial_{2}^{2}+\left(y_{2}+b_{2} c_{2}\right) \partial_{1}-\left(y_{1}+b_{1} c_{1}\right) \partial_{2}, \\
& E^{\prime}=r \partial_{r}-2\left(x_{11} \partial_{1}^{2}+x_{12} \partial_{1} \partial_{2}+x_{22} \partial_{2}^{2}\right) \\
& \left.\quad-\left(\left(y_{1}+b_{1} c_{1}\right) \partial_{1}+\left(y_{2}+b_{2} c_{2}\right) \partial_{2}\right)-1-d^{3}\right\rangle .
\end{aligned}
$$

We note that $\operatorname{rank}(I)=\operatorname{rank}\left(I^{\prime}\right)$ holds for any set of values of the constants.

Secondly, we calculate in $(-w, w)\left(I^{\prime}\right)$ for the weight vector $w=(0,1,0,0,0,0)$ where each weight stands for the variables $\partial_{11}, \partial_{12}, \partial_{22}, \partial_{1}, \partial_{2}, \partial_{r}$ respectively. In order to perform Buchberger's algorithm with respect to the $(-w, w)$-weight 
order, we need to consider the homogenized system $I^{\prime h}$ for $I^{\prime}$ :

$$
\begin{aligned}
I^{\prime h}=\left\langle A_{11}^{\prime h}\right. & =h x_{11} \partial_{11}-x_{11} \partial_{1}^{2}-\underline{a_{11}^{3}}, A_{12}^{\prime h}=h x_{12} \partial_{12}-x_{12} \partial_{1} \partial_{2}-\underline{a_{12}^{3}}, \\
& A_{22}^{\prime h}=h x_{22} \partial_{22}-x_{22} \partial_{2}^{2}-\underline{a_{22}^{3}}, \\
B= & \partial_{1}^{2}+\partial_{2}^{2}-\underline{r^{2}} \\
C_{12}^{\prime h} & =x_{12} \partial_{1}^{2}+2\left(x_{22}-x_{11}\right) \partial_{1} \partial_{2}-x_{12} \partial_{2}^{2}+\left(y_{2} h+b_{2} c_{2}\right) \partial_{1}-\left(y_{1} h+\underline{\left.b_{1} c_{1}\right) \partial_{2}},\right. \\
E^{\prime h} & =h r \partial_{r}-2\left(x_{11} \partial_{1}^{2}+x_{12} \partial_{1} \partial_{2}+x_{22} \partial_{2}^{2}\right) \\
& \left.\quad-\left(\left(h y_{1}+b_{1} c_{1}\right) \partial_{1}+\left(h y_{2}+b_{2} c_{2}\right) \partial_{2}\right)-h^{3}-\underline{d^{3}}\right\rangle .
\end{aligned}
$$

We denote by $<_{(-w, w, 0)}^{h}$ an order in the homogenized Weyl algebra which compares the total degree first, $(-w, w, 0)$-degree second, otherwise we apply the following block order as a tie breaker: $d \gg r \gg\left\{a_{11}, a_{12}, a_{22}\right\} \gg\left\{b_{1}>\right.$ $\left.b_{2}\right\} \gg\left\{c_{1}, c_{2}\right\} \gg\left\{y_{1}, y_{2}\right\} \gg \partial_{r} \gg\left\{\partial_{12}\right\} \gg\left\{\partial_{11}, \partial_{22}\right\} \gg\left\{\partial_{1}, \partial_{2}\right\} \gg\left\{x_{12}\right\} \gg$ $\left\{x_{11}, x_{22}\right\} \gg h$. Here, the symbol $>$ represents the lexicographic order. The underlined parts in $I^{\prime h}$ are initial terms with respect to $<_{(-w, w, 0)}^{h}$. They are pairwise coprime and their commutators are equal to zero except $\left[B, E^{\prime h}\right]=$ $-2 h B$. From Lemma 1 we conclude that the set $\left\{A_{11}^{\prime h}, A_{12}^{\prime h}, A_{22}^{\prime h}, B, C_{12}^{\prime h}, E^{\prime h}\right\}$ is a Gröbner basis of $I^{\prime h}$ in $D^{h}\left[a_{11}, a_{12}, a_{22}, b_{1}, b_{2}, c_{1}, c_{2}, d\right]$ with respect to $<_{(-w, w, 0)}^{h}$. In other words, the transformation of dependent and independent variables gives us the Gröbner basis without adding new elements. Dehomogenizing $I^{\prime h}$, we obtain

$$
\begin{aligned}
\operatorname{in}_{(-w, w)}\left(I^{\prime}\right)= & \left\langle\tilde{A}_{11}^{\prime}=x_{11} \partial_{11}-x_{11} \partial_{1}^{2}-a_{11}^{3}, \tilde{A}_{12}^{\prime}=x_{12} \partial_{12}-a_{12}^{3},\right. \\
& \tilde{A}_{22}^{\prime}=x_{22} \partial_{22}-x_{22} \partial_{2}^{2}-a_{22}^{3}, \\
& B=\partial_{1}^{2}+\partial_{2}^{2}-r^{2}, \\
& \tilde{C}_{12}^{\prime}=2\left(x_{22}-x_{11}\right) \partial_{1} \partial_{2}+\left(y_{2}+b_{2} c_{2}\right) \partial_{1}-\left(y_{1}+b_{1} c_{1}\right) \partial_{2}, \\
& \tilde{E}^{\prime}=r \partial_{r}-2\left(x_{11} \partial_{1}^{2}+x_{22} \partial_{2}^{2}\right) \\
& \left.\quad-\left(\left(y_{1}+b_{1} c_{1}\right) \partial_{1}+\left(y_{2}+b_{2} c_{2}\right) \partial_{2}\right)-1-d^{3}\right\rangle .
\end{aligned}
$$

In this calculation, we regard $a_{11}, a_{12}, a_{22}, b_{1}, b_{2}, c_{1}, c_{2}, d$ as ring variables with the weight 0 . As in the proof of Theorem 3, the equation above holds when $a_{11}, \ldots, d$ are specialized to generic complex numbers. The holonomic rank of the $(-w, w)$-initial ideal $\tilde{I}^{\prime}:=\operatorname{in}_{(-w, w)}\left(I^{\prime}\right)$ coincides with that of the diagonal system transformed by the same change of variables for $I^{\prime}$ from $I$. The holonomic rank of $\tilde{I}^{\prime}$ agrees with that of

$$
\begin{aligned}
\tilde{I}=\langle & \left\langle\tilde{A}_{11}=\underline{\partial_{11}}-\partial_{1}^{2}, \tilde{A}_{12}=\underline{\partial_{12}},\right. \\
& \tilde{A}_{22}=\underline{\partial_{22}}-\partial_{2}^{2}, \\
& B=\underline{\partial_{1}^{2}}+\partial_{2}^{2}-r^{2}, \\
& \tilde{C}_{12}=2\left(x_{22}-x_{11}\right) \underline{\partial_{1} \partial_{2}}+y_{2} \partial_{1}-y_{1} \partial_{2}, \\
& \left.\tilde{E}=r \underline{\partial_{r}}-2\left(x_{11} \partial_{1}^{2}+x_{22} \partial_{2}^{2}\right)-\left(y_{1} \partial_{1}+y_{2} \partial_{2}\right)-1\right\rangle .
\end{aligned}
$$


Hence, we obtain the following inequality:

$$
\operatorname{rank}(I)=\operatorname{rank}\left(I^{\prime}\right) \geq \operatorname{rank}\left(\operatorname{in}_{(-w, w)}\left(I^{\prime}\right)\right)=\operatorname{rank}\left(\tilde{I}^{\prime}\right)=\operatorname{rank}(\tilde{I}) .
$$

Finally, we show that $\operatorname{rank}(\tilde{I})=4$. Proposition 1 tells us that the set

$$
\begin{aligned}
& \left\{\tilde{A}_{11}, \tilde{A}_{12}, \tilde{A}_{22}, B, \tilde{C}_{12}, \tilde{E}\right. \\
& \left.\quad D_{2}=2\left(x_{22}-x_{11}\right)\left(\underline{\partial_{2}^{3}}-r^{2} \partial_{2}-\frac{y_{2} \partial_{1}^{2}-y_{1} \partial_{1} \partial_{2}-\partial_{2}}{2\left(x_{22}-x_{11}\right)}\right)\right\} .
\end{aligned}
$$

is a Gröbner basis of $R \tilde{I}$ with respect to the block order $\left\{\partial_{r}\right\} \gg\left\{\partial_{11}>\partial_{22}\right\} \gg$ $\left\{\partial_{1}>\partial_{2}\right\}$, where the tie breaker $>$ represents the graded lexicographic order. The set of standard monomials is $\left\{1, \partial_{1}, \partial_{2}, \partial_{2}^{2}\right\}$. It means that the holonomic rank of $\tilde{I}$ is 4 .

\section{References}

[1] T. Koyama, A Holonomic Ideal Annihilating the Fisher-Bingham Integral, to appear in Funkcialaj Ekvacioj.

[2] T. Koyama, H. Nakayama, K. Nishiyama, N. Takayama, Holonomic Gradient Descent for the Fisher-Bingham Distribution on the $d$-dimensional Sphere, http://arxiv.org/abs/1201.3239

[3] E. Miller, L. Matusevich, U. Walther, Homological Methods for Hypergeometric Families, Journal of the American Mathematical Society 18 (2005), 919-941.

[4] H. Nakayama, K. Nishiyama, M. Noro, K. Ohara, T. Sei, N. Takayama, A. Takemura, Holonomic Gradient Descent and its Application to the Fisher-Bingham Integral, Advances in Applied Mathematics 47 (2011), 639-658.

[5] T. Oaku, N. Takayama, Algorithms for D-modules - Restriction, Tensor Product, Localization, and Local Cohomology Groups, Journal of Pure and Applied Algebra 156 (2001), 267-308.

[6] M. Saito, B. Sturmfels, N. Takayama, Gröbner Deformations of Hypergeometric Differential Equations, Springer, 2000. 